\title{
Review Article \\ Mesenchymal Stem/Stromal Cells in Stromal Evolution and Cancer Progression
}

\author{
Francesca Cammarota and Mikko O. Laukkanen \\ IRCCS SDN, Via Emanuele Gianturco 113, 80431 Naples, Italy \\ Correspondence should be addressed to Mikko O. Laukkanen; mlaukkanen@sdn-napoli.it
}

Received 29 June 2015; Revised 27 August 2015; Accepted 1 September 2015

Academic Editor: Eva Mezey

Copyright ( 2016 F. Cammarota and M. O. Laukkanen. This is an open access article distributed under the Creative Commons Attribution License, which permits unrestricted use, distribution, and reproduction in any medium, provided the original work is properly cited.

\begin{abstract}
The study of cancer biology has mainly focused on malignant epithelial cancer cells, although tumors also contain a stromal compartment, which is composed of stem cells, tumor-associated fibroblasts (TAFs), endothelial cells, immune cells, adipocytes, cytokines, and various types of macromolecules comprising the extracellular matrix (ECM). The tumor stroma develops gradually in response to the needs of epithelial cancer cells during malignant progression initiating from increased local vascular permeability and ending to remodeling of desmoplastic loosely vascularized stromal ECM. The constant bidirectional interaction of epithelial cancer cells with the surrounding microenvironment allows damaged stromal cell usage as a source of nutrients for cancer cells, maintains the stroma renewal thus resembling a wound that does not heal, and affects the characteristics of tumor mesenchymal stem/stromal cells (MSCs). Although MSCs have been shown to coordinate tumor cell growth, dormancy, migration, invasion, metastasis, and drug resistance, recently they have been successfully used in treatment of hematopoietic malignancies to enhance the effect of total body irradiation-hematopoietic stem cell transplantation therapy. Hence, targeting the stromal elements in combination with conventional chemotherapeutics and usage of MSCs to attenuate graft-versus-host disease may offer new strategies to overcome cancer treatment failure and relapse of the disease.
\end{abstract}

\section{Introduction}

Tumors are organ-like structures [1] composed of numerous cell types whose interactions are required to drive and promote their growth and metastasis [2,3]. Carcinogenic cells recruit nontumorigenic cells both locally from the neighboring tissues as well as from the circulation to construct the tumor microenvironment, which through reciprocal cancerstroma interactions coevolves to promote cancer progression through paracrine signaling and physical interactions [48]. The tumor microenvironment contains cancer-associated fibroblasts (CAFs) [2], endothelial cells [9, 10], immune cells [11, 12], adipocytes [13], cancer stem cells (CSC) that differentiate into metastatic epithelial cells $[14,15]$, mesenchy$\mathrm{mal}$ stem/stromal cells (MSCs) that can differentiate into fibroblasts and other types of cells representing mesenchymal lineages [16], and various types of extracellular matrix (ECM) proteins [3] needed for reciprocal messaging and the stimulation of tumor growth. The stroma, especially MSCs and stromal cells originating from MSCs, has recently been recognized as a player in carcinogenesis, affecting tumor growth, development, and progression beginning at the early steps of tumorigenesis [4] and influencing the construction of the microenvironment, epithelial mesenchymal transition, and metastasis, that is, functions that are essential for tumor maintenance and metastasis to other tissues [17-21].

\section{Evolution of the Tumor Stroma}

Simultaneous with the changes causing the immortalization of epithelial cells, there is a gradual evolution of the tumor microenvironment that includes (i) increased local vascular permeability; (ii) the extravasation of plasma and macromolecules, such as fibrinogen and plasminogen; (iii) the activation of coagulation mechanisms in the developing tumor microenvironment; (iv) the formation of fibrin gel deposits; (v) the formation of a provisional stroma comprising cancer 
cells, fibroblasts, and immune cells; (vi) the initiation of angiogenesis in the provisional stroma; (vii) the degradation and replacement of the provisional stroma fibrin with highly vascularized granulation connective tissue; (viii) the transformation of the stroma to desmoplastic, loosely vascularized, and dense connective tissue; and (ix) the remodeling of the stromal ECM, inducing local cancer cell migration and metastasis [22-26].

2.1. Increased Vascular Permeability. MSCs may contribute to increased vascular permeability alone or by attracting mast cells that are able to both initiate and sustain cellular trafficking. Increased vascular endothelial growth factor-A (VEGF-A) production is one of the main drivers of vascular hyperpermeability $[27,28]$. VEGF-A binding to VEGF receptor 2 (VEGFR2) induces a conformational change and subsequent dimerization of the receptor, leading to autophosphorylation and initiation of downstream signal transduction [29]. The activated signal transduction leads to increased vascular permeability through two alternative mechanisms: by the synthesis of transcellular endothelial pores or by the transient opening of paracellular endothelial junctions. The action of VEGF depends on reactive oxygen and nitrogen species (ROS, RNS), the activation of the SRC family of protooncogenes, and their contact with adherens junction VE-cadherin proteins [28,30]. According to recent reports, mast cells contribute to vascular permeability by secreting histamine, serotonin, and platelet-activating factor that activate TR3/Nur77 orphan nuclear transcription factor signaling. TR3/Nur77 increases vascular permeability by suppressing the expression of endothelial cell adherent junctionassociated proteins (VE-cadherin, $\beta$-catenin, $\gamma$-catenin, and p120) and tight junction protein CLAUDIN 5 that maintains vascular homeostasis [31,32]. Alternatively, mast cell secreted histamine induces vascular permeability by nitric oxide(NO-) dependent vascular dilation and $\mathrm{PKC} / \mathrm{ROCK} / \mathrm{NO}-$ dependent endothelial barrier disruption or by binding to H1 G-protein coupled receptor that activates endothelial cell calcium influx enhancing vascular permeability related signal transduction $[33,34]$.

\subsection{Development of the Fibrin Matrix-Derived Provisional} Stroma into Mature Stroma. The extravasation of plasma components, such as fibrinogen and clotting proteins (prothrombin and factors V, VII, X, and XIII), through the endothelial cell layer is one of the earliest changes in the precancerous lesion environment and initiates a tissue response similar to wound healing $[22,24,27,34,36-40]$. The synthesis of fibrin from fibrinogen by the action of thrombin demarcates the first milestone in tumor stroma development [22, 27, $36,39,41,42]$. Thrombin cleaves fibrinogen into soluble fibrin monomers and activates the clotting factor XIII to factor XIIIa, which then converts the soluble fibrin monomers into insoluble fibrin polymers to form a cross-linked fibrin matrix [43]. The formation of the fibrin matrix dramatically changes the tissue composition by creating a gel that absorbs and arrests plasma solutes, resulting in tissue edema. Therefore, the fibrin gel forms an initial "provisional" stroma where the parenchymal tumor cells, mesenchymal stromal cells, and hematopoietic inflammatory cells can migrate to comprise the final tumor microenvironment [44]. Fibrin gel itself enhances angiogenesis, another early tumor stroma phenomenon, by protecting angiogenic growth factors from degradation, by inducing the production of proangiogenic molecules, and by directly creating angiogenic factors, such as fragment $\mathrm{E}$ [45]. The next phase in the development of the tumor microenvironment and the formation of the mature tumor stroma is a result of the coordinated actions of infiltrated macrophages and fibroblast-derived proteases that degrade the "provisional" stroma fibrin, replacing it with loose connective tissue $[22,39]$. The loose connective tissue, which resembles the granulation tissue in healing wounds, stimulates the growth of fibroblasts and new blood vessels.

2.3. Development of Stromal Desmoplasia. One of the histological cornerstones of cancer development is the formation of a dense fibrotic stromal matrix comprising ECM and activated fibroblasts (myofibroblasts). In this last phase of stromal development, the granulation tissue transforms into desmoplastic dense connective tissue characterized by poor vascularization. The activation of the stroma, desmoplasia, can be interpreted as an attempt by the tumor tissue to heal the injury produced by the infiltrative and destructive growth of cancer cells, indicating the invasive and malignant characteristics of the tumor. However, it has been suggested that the increased collagen synthesis in desmoplasia, together with myofibroblast-induced tissue retraction, may paradoxically constitute a protective mechanism with invasive characteristics [10]. Mechanistically, the desmoplastic response is a poorly understood process associated with invasive tumors, such as diffuse infiltrative pancreatic and gastric carcinomas and infiltrating ductal (scrirrhous) carcinomas of the breast, and involves the excessive production of types I and III collagens and elastin [10]. In scrirrhous carcinomas of the breast, myofibroblasts, together with fibrin and collagen III, are mostly present in the immature mesenchymal stroma at the tumor periphery, while collagen-I is expressed in the mature sclerotic connective tissues of the tumor center [11].

In several types of tumors, such as thyroid cancer, the desmoplastic stromal reaction is a relatively common phenomenon, being present in up to $80 \%$ of medullary thyroid cancers and correlating with lymph node metastasis [46]. Indeed, the activated stroma has been considered a marker of invasion and metastatic cancer development. In thyroid cancers, desmoplasia induces increased production of collagen by stromal fibroblasts more prominently in anaplastic thyroid and medullary thyroid cancer than in papillary thyroid cancer, directly correlating with increased aggressiveness and lymph node metastasis [46]. It is therefore used as an intraoperative prognostic marker $[47,48]$. The desmoplastic stroma in thyroid cancer contains activated fibroblasts (cancer-associated fibroblasts (CAFs) or myofibroblasts) that, together with the other stromal components, initiate the remodeling of the extracellular matrix [49]. ROS have been shown to promote the activation of these fibroblasts, which 
then increase tumor cell proliferation, tumor-associated inflammation, and angiogenesis by expressing invasionassociated factors and enzymes, such as fibroblast activation protein $\alpha(\mathrm{FAP} \alpha)$ and matrix metalloproteases (MMP), which are able to degrade and remodel the ECM [50-52].

2.4. Remodeling of the Stromal ECM Facilitates the Migration of Cancer Cells. The remodeling of the ECM induces the migration, invasion, and metastasis of cells by stimulating epithelial cell transformation and local migration by altering the cross-linked stromal structure. It therefore represents a critical element of cancer progression. Proteolytic degradation of the ECM affects the integrinmediated anchorage of the cells, focal adhesions at cell membranes, cellular cytoskeletal organization, and the signal transduction regulating these structures. One of the main nonreceptor tyrosine kinases affected during this process is focal adhesion kinase (FAK), which directly signals through the SRC oncogene family kinases, linking integrin signaling to RAS-BRAF-MEK-ERK mitogen pathway signaling, thus inducing the malignant progression of tumor cells [53, 54].

\section{MSCs and CAFs in the Tumor Stroma}

In tumors, the MSCs either differentiate or maintain their primitive phenotype, thus participating in the construction of the stroma and supporting cancer cell growth through their secretion of cytokines and growth factors [55-58]. The source of tumor MSCs has not been completely clarified, although they have been isolated from most tissues [59], suggesting that they have a local origin. Another potential source is the circulating bone marrow-derived MSCs that extravasate from the circulation and then home and engraft onto growing tumors [60-62]. Evidence of the tumor tropism of MSCs has been obtained from in vivo studies, in which transplanted MSCs were demonstrated to migrate to tumors [60]. Interestingly, the migration of MSCs to tumors seems to be increased by cell damage, which typically characterizes the clinical treatments used for cancer, such as radiation therapy, possibly due to the increased cytokine and chemokine secretion from the injured tissues [61]. Although the detailed mechanism(s) underlying the migration and homing of MSCs to tumors are not well documented, MSCs have a similar homing mechanism to inflammatory cells, hematopoietic stem cells, and cancer cells in that they utilize the same adhesion molecules and cytokines/chemokines, most notably the CXCR4-CXCL12 receptor-ligand binding system $[63,64]$.

Cancer-associated fibroblasts (CAFs) are derived from both mesenchymal stem/stromal cells and local fibroblasts $[60,65]$, thus supporting observations suggesting the presence of primitive undifferentiated MSCs even in advanced cancers. MSCs and CAFs are both heterogeneous populations that may have different phenotypic characteristics even within the same type of cancer [66, 67]. CAFs are a rich source of growth promoting molecules (e.g., HGF, LOXL2, and TENASCIN-C) and proangiogenic factors (e.g., VEGF), hence playing an important role in cancer progression and metastasis [65] by stimulating epithelial mesenchymal transition (e.g., by TWIST1 and SNAIL production), by causing epigenetic changes, and by altering three-dimensional structure of ECM (e.g., by MMP and plasminogen activator protein production) $[67,68]$. CAFs are known to support survival and proliferation of cancer cells in metastasis in a similar mechanism as in primary tumor. An interesting characteristic of CAFs is their ability to migrate together with epithelial cancer cells, thus suggesting a role in the intravasation and extravasation of epithelial cells in metastasis process by promoting cancer cell transmigration through endothelial cell layers $[69,70]$, hence supporting the hypothesis that primary tumor may be able to facilitate metastasis by providing the microenvironment.

Previous papers have demonstrated that in certain cases CAFs, similarly with MSCs, maintain normal fibroblast tumor suppressive characteristics. Receptor-ligand ROBO1SLIT2 cancer-stroma interaction has been shown to reduce tumor cell proliferation by reducing PI3K- $\beta$-catenin and SDF1-CXCR4 signal transduction and consequent cancer cell malignancy [71, 72]. Interestingly, aggression of cancer cells lacking $\mathrm{ROBO} 1$ receptor molecule was increased by CAFs expressing SLIT1 ligand [73], whereas RNAi SLIT1 increased hepatocyte growth factor-mediated cancer cell migration and invasion by upregulating CDC42 Rho GTPase activity [74], thus giving more insight into the inhibitorystimulatory mechanism of CAFs that may depend on phenotypic differences of stromal cells and malignant epithelial cells. The study of Takahashi and coworkers corroborated selective tumor suppressor properties of CAFs. According to their observations, podoplanin positive CAFs predicted poor survival and outcome among lung adenocarcinoma and squamous cell carcinoma patients, whereas in small cell lung cancer podoplanin positivity suggested better prognosis [75]. While the characteristics of MSCs and CAFs are well documented in tumor support, more work is needed to study differentiation of MSCs to cancer-associated fibroblasts and tumor suppressor properties of these cell populations.

3.1. Tumor MSCs Differ from Normal MSCs. It is important to note that the MSCs and CAFs localized in the tumor stroma have a different phenotype compared to MSCs and fibroblasts isolated from normal tissues, which may be a result of the constant exposure of these cells to inflammatory and cancer cell-secreted cytokines inducing procancerous characteristics [76-78]. Although the tumor-associated MSCs share similar cell surface markers and functionality with normal tissue MSCs, the paracrine effect on cancer cell proliferation is different [58]. Whereas normal tissue MSCs have been suggested to reduce inflammatory and cancer cell proliferation $[79,80]$, tumor-associated MSCs increase their growth [5355]. The phenotypic and functional modifications of MSCs are further supported by case reports demonstrating unusual papillary thyroid carcinoma- and lung carcinoma-associated intratumoral heterotrophic ossification $[81,82]$. However, the heterotopic ossification caused by the differentiation of MSCs into osteoprogenitor cells and further differentiated cells [83] 
may also be the result of abnormal differentiation signaling originating from the surrounding tumor stroma.

\subsection{MSCs Support Parenchymal Cells in the Tumor Microenvi-} ronment. Most transformed cell lines are not able to survive after transplantation and are therefore considered to be cells continuously growing without tumorigenic characteristics. Even among highly carcinogenic cell lines, only a small subset harboring stem cell-like characteristics are able to initiate tumor growth in vivo [84]. Due to their phenotype and functional properties, cells with clonal tumor-initiating capacity are called cancer stem cells (CSCs) or tumor-initiating cells (TICs). The CSCs/TICs reside in specific niches in the tumor microenvironment that maintain their plasticity, protect them from immune defense mechanisms, and modify their metastatic potential. MSCs have been shown to interact with CSCs/TICs, supporting parenchymal cell growth and causing increased resistance to therapy [35], cancer cell dormancy, and evasion from the immune system [85] either through paracrine secretion $[35,57]$ or gap junction contact [85]. Alternatively, MSCs can affect epithelial cancer cell function by direct contact, causing increased expression of microRNAs, such as mir199a and stem cell-associated factors, in the epithelial cells [86]. MSCs secrete various growthsupporting cytokines, growth factors, and microRNAs that in some cases are stored inside extracellular vesicular particles (exosomes) [87]. Exosomes are small $(40-100 \mathrm{~nm}$ in diameter) membrane-bound organelles that function as part of an intercellular communication mechanism. During tumorigenesis, exosome-bound factors have been demonstrated to modify the phenotype of the epithelial cancer cells or tumor stromal cells to support the aggressive phenotype and tumor progression. The exosomes characteristically include various types of molecules, including matrix metalloproteases [88], platelet-derived growth factors [89], molecules that activate signal transduction [90], oncomiRs, bioactive lipids, and metabolites [91].

The gap junctions between stromal cells and parenchymal cells are an important gateway for microRNA transfer. Interestingly, gap junction-transferred CXCL12-targeting microRNAs mir127, mir197, mir222, and mir223, can reduce cancer cell proliferation and even induce dormancy that may last for decades, eventually leading to a relapse of the disease due to bone marrow metastasis [91]. A recent study demonstrated that there was gap junction-mediated intercellular communication between bone marrow MSCs and primitive Oct4-expressing breast cancer tumor-initiating cells [91], suggesting that there is a preference for gap junction-mediated connections between cells harboring stem cell characteristics, also suggesting that these cells show similarities to hematopoietic stem cell dormancy [92, 93].

In addition to the gap junction structures, it has been suggested that breast cancer cells form hybrids with MSCs [94]. Recently, a number of publications have suggested that there is a direct connection between MSCs and cancer cells, resulting in increased epithelial cell growth and survival $[55,95-$ 99]. The direct connection between cancer cells and stromal cells has been studied in a cancer cell-stromal cell coculture system that could itself promote cell-to-cell contacts. Characteristically, primary nontransformed cells avoided direct contact with other cells, limiting their growth and migration, a phenomenon known as contact inhibition [100]. We have studied the interaction of transformed cells and human bone marrow-derived mesenchymal stem cells and demonstrated that physical MSC-MSC or MSC-cancer cell interaction is mediated through temporal membrane protrusions, which direct the movement of the cells (Supplemental film 1 in Supplementary Material available online at http://dx.doi.org/ 10.1155/2016/4824573) [97]. Unlike primary cells, cancer cells have lost the contact inhibition phenotype [101] and therefore characteristically create network connections by reaching towards other cancer cells and nontransformed primary cells, such as MSCs (Supplemental film 2). The aggression of cancer cells in coculture can induce damage to the MSCs and activate apoptosis, with the consequent loss of MSCs locomotion [97]. The damaged stromal cells that are immobilized and unable to avoid contact are vulnerable to cancer cell aggression, resulting in material transfer from stromal cells to epithelial cancer cells (Figure 1) [97, 99]. Consequently, the tumor initiating and prometastasis effects of the transformed cells increase significantly, and this is linked to increased mitochondrial activity. Importantly, we demonstrated by serial dilution transplantation and a flow cytometric analysis of clusters of differentiation markers that the transformed cells did not show any stem cell characteristics, suggesting that increased metabolic activity mediates the aggressiveness of the epithelial cells. Interestingly, a cell-count analysis of the transformed cells suggested that there was only minor growth during cytoplasmic material transfer, whereas control cells grown in the absence of damaged MSCs showed continuous cell proliferation (Figure 2) [97]. Thus, these observations may suggest that cancer cells can remove injured and dying tumor cells, thereby temporally increasing their own growth characteristics. This hypothesis is corroborated by findings suggesting that there was increased mouse tumor cell growth in the presence of apoptotic cancer cells or fibroblasts, underlining the importance of cellular damage in direct cellto-cell contact and the transfer of cellular macromolecules $[55,97,102,103]$.

In addition to the paracrine-mediated, gap junctionmediated, and direct contact-based support of tumorigenesis, stromal cells have been shown to affect the transcriptome of tumors, especially in patients with a relapse of the disease. The dataset analyses have demonstrated upregulation of the gene expression patterns associated with poor patient survival [1921]. Analyses have shown that colon cancer patients had significantly higher expression levels of genes related to the risk of recurrence, such as TGF- $\beta, C A L D 1, P O S T N, F A P$, $I G F B P 7$, and $M P G$, in their stromal cells compared to their epithelial cancer cells $[19,20]$. Notably, among all stromal cell populations, the CAFs showed the strongest expression level of the stem/serrated/mesenchymal transcription subtype of colorectal cancer [20]. Thus, the "stromal signatures" characteristic of different colorectal cancer subtypes may have clinical relevance and may even serve as a prognostic marker of the disease [20]. 

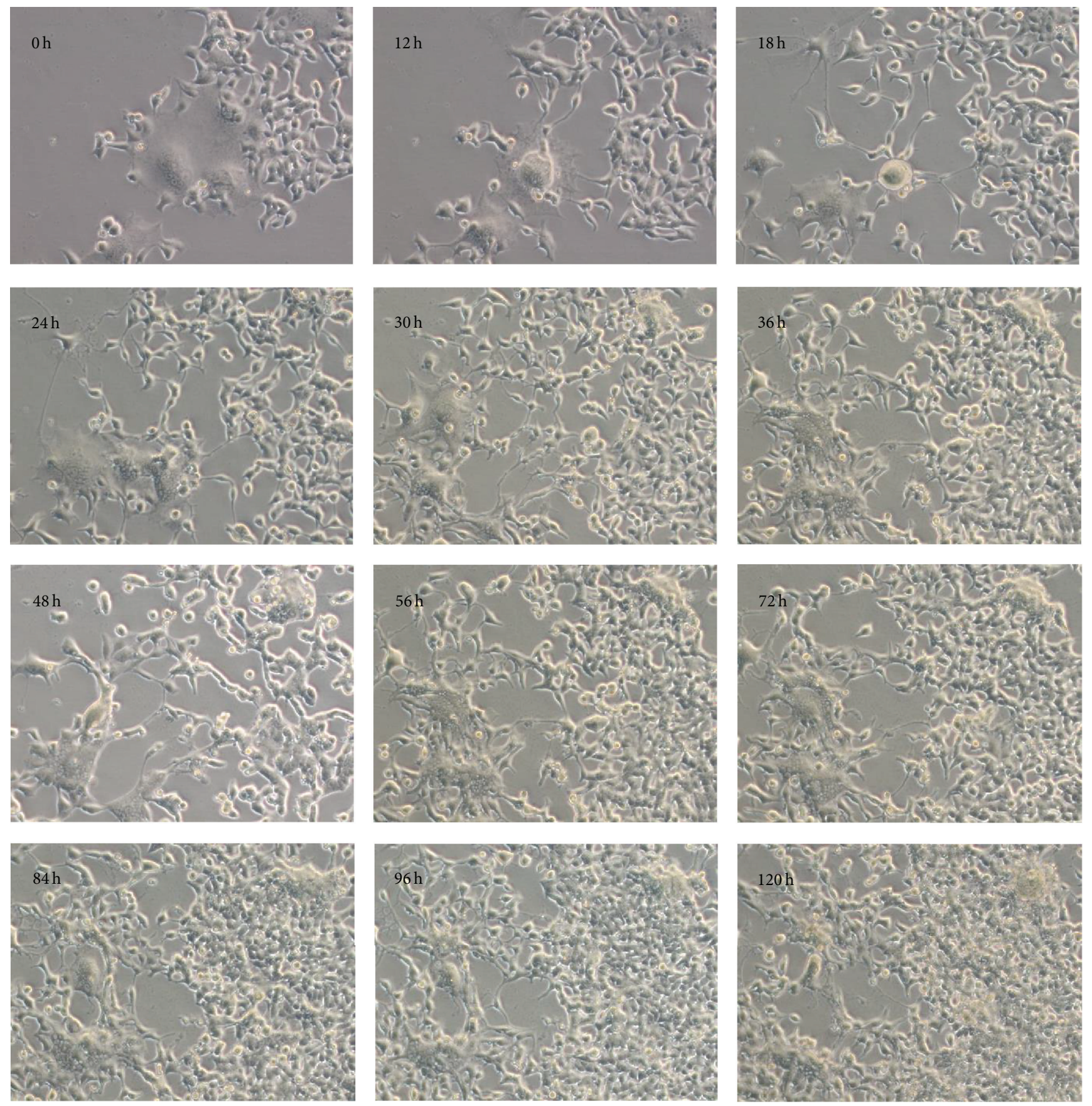

FIgURE 1: Time-lapse images of HEK 293T and bone marrow mesenchymal stem cell culture [35]. Apoptotic MSCs were surrounded and consumed by the HEK 293 T cells during the 120 -hour period.

\section{MSCs in Cancer and in Graft-versus-Host Disease Therapy}

Although preclinical cancer therapy studies in MSC xenograft mouse models have given contradictory results, which may be due to model systems used in the experiments, timing of MSC transplantation, and protocol of propagating cells ex vivo [104], MSCs have maintained their therapeutic potential in cancer treatments. In ongoing phase I/II study (NCT02008539) autologous MSCs transduced with herpes simplex virus-thymidine kinase (HSV-TK) retrovirus are intravenously injected into patients with advanced gastrointestinal tumors followed by ganciclovir treatment to study safety and tolerability of the therapy [105]. Significantly more excitement has received the property of MSCs to enhance hematopoietic stem cell engraftment and to prevent graft-versus-host disease (GVHD). GVHD, the attack of transplanted immune cells against recipient tissues, is a frequent complication of autologous bone marrow transplantation in the treatment 


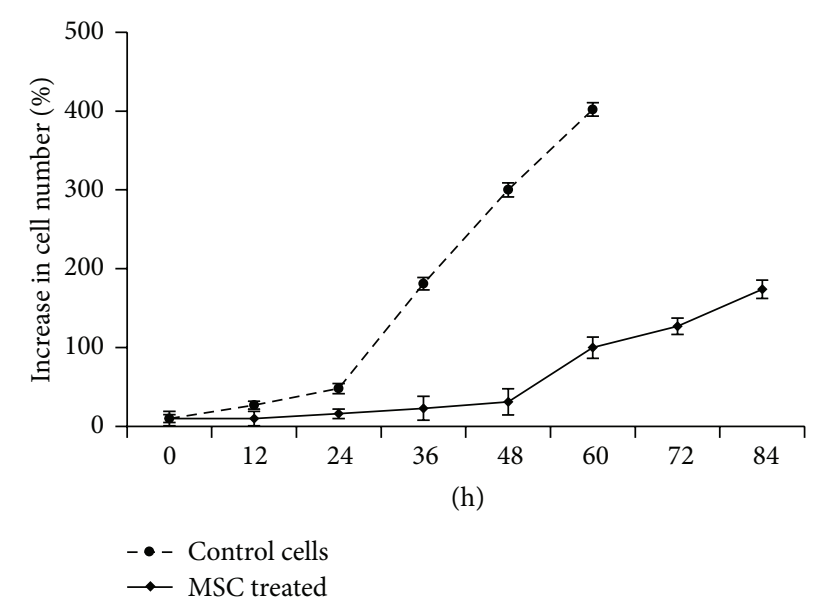

FIGURE 2: The results of cell count analyses of HEK 293T cells grown in the absence (control cells) or presence of apoptotic MSCs (MSCtreated) [35]. The analyses suggest that there was decreased growth of the MSC-treated cells during 0-48 hours of cytoplasmic material transfer from the MSCs to HEK 293T cells.

of hematologic malignancies. MSC transplantation has been demonstrated to be safe in a clinical pilot study in which twenty patients with hematologic malignancies received MSC transplantation together with allogeneic hematopoietic cell transfusion. The cotransplantation resulted in $10 \%$ one-year nonrelapse mortality, $80 \%$ overall survival, $60 \%$ progression-free survival, and $10 \%$ graft-derived death. Similar treatments without MSC cotransplantation have resulted in $37 \%$ one-year nonrelapse mortality, $44 \%$ overall survival, $38 \%$ progression-free survival, and $31 \%$ graftderived death. In another clinical trial, the effect of MSC transfusion was studied in 75 patients with persistent acute GVHD that did not respond to immunosuppressive agents. The GVHD status before MSC treatments was improving in $2.7 \%$ of patients, was unchanged in $29.3 \%$ of patients, and was worsening or showing maximal GVHD level in $66.7 \%$ of patients. Serial MSC transfusions demonstrated an encouraging $61.3 \%$ overall response rate that was followed by $78.1 \% 100$-day survival level, whereas patients who did not responded to MSC infusions had 100-day survival of only $31 \%$. The ex vivo culture of MSCs before transfusion decreases the overall survival of the patients although the phase I/II clinical trials have demonstrated safety of the procedure [106-109]. Currently new clinical trials (EudraCT number 2006-004101-26, EudraCT number 2009-014980-38, NCT01763099, NCT01763086, and NCT01941394) are recruiting patients to further explore the ability of MSCs to prevent GVHD. Hence, based on the observed data and ongoing studies, MSCs transplantation is a promising tool to improve the efficiency of cancer treatments.

\section{Tumor Stroma as a Drug Target and Mediator of Drug Resistance}

Molecular-targeting drugs against activated oncogenes whose continues expression is essential for the survival of cancer cells, a phenomenon known as oncogene addiction, represent the latest development in cancer treatment. However, the efficacy of these therapies is reduced by the development of drug resistance. Cell autonomous drug resistance, also known as primary resistance, is caused by the constitutive activation (mutation) of signal transducers downstream of the targeted molecule or by the simultaneous activation of compensatory pathways. Secondary or acquired resistance is observed when neoplastic cells originally sensitive to molecular inhibitors lose their response to these drugs. This can occur by target reactivation or by compensatory bypass. The cell autonomous mechanism implies that there are secondary mutations in the oncogenic kinase that render it refractory to the treatment. In contrast, compensatory bypass involves the compensatory activation of alternative kinases, thereby reducing the biological effects of the drug [110].

Together with the cell autonomous mechanisms of resistance to molecular inhibitors, many studies have suggested that an important role is played by noncell autonomous signals, originated by the cellular components of the tumor microenvironment [110]. A striking feature of metastatic tumor cells is their ability to plastically adapt to diverse microenvironmental conditions and to overcome single-drug treatment. The mechanisms underlying this mode of drug resistance are largely elusive and have thus been the subject of intense preclinical investigation. Recent studies have highlighted a crucial role for the interaction between cancer cells and the tumor microenvironment, leading investigators to hypothesize a completely different and unconventional mechanism of resistance to molecular inhibitors [97, 99, 103]. Although these studies were conducted in coculture systems, they form a proof of principle, demonstrating a novel mechanism by which cancer cells can survive under extreme stress and even utilize the severe culture conditions to improve their growth characteristics.

Poorly vascularized, desmoplastic stroma supports tumorigenesis and simultaneously forms a barrier for chemotherapeutic drugs, making it as an attractive drug target. Combination treatments targeting both the stroma and cancer cells with cytotoxins have shown promising results in preclinical and clinical experiments. A complete depletion of stroma by IPI-926 and a sonic hedgehog inhibitor, together with gemcitabine administration, increased the drug delivery in a preclinical mouse model, thus underlining the importance of the stroma for tumor development and maintenance and further suggesting the efficacy of combination therapy that targets both cancer cells and tumor stromal components [111]. A clinical study with partial stromal depletion using CD40-activated macrophages as well as a preclinical work utilizing enzymatic depletion of ECM hyaluronan demonstrated improved patient survival and increased drug delivery into the tumor [112]. In addition to the destruction of the whole stroma, preclinical studies have shown reduced tumorigenesis after depletion of stromasupporting myofibroblasts using antibodies targeted against fibroblast activation protein (FAP) [113]. Clinical studies have demonstrated safety of anti-FAP infusion into patients with colorectal carcinoma, metastatic colorectal cancer, and small cell lung cancer although no tumor response has been 
observed $[114,115]$. Despite poor success in cancer therapy, a recent anti-FAP preclinical study of malignant pleural mesothelioma, an incurable disease resulting from exposure to asbestos, suggested lysis of FAP positive cells, inhibited tumor growth, and significantly prolonged the survival of the mice [116]. The data encouraged the research team from the University of Zurich Switzerland to open a new clinical phase I trial (NCT01722149) to study the effect of FAP-specific CD8 positive $\mathrm{T}$ cells in malignant pleural mesothelioma patients. In the trial the patients are infused with $1 \times 10^{6}$ adoptively transferred FAP-specific retrovirally reprogrammed $\mathrm{T}$ cells directly in the pleural effusion to evaluate the safety and the efficacy of the immunotherapy. Besides antibodies FAP vaccination studies have resulted in some excitement in preclinical level demonstrating significantly reduced tumor growth and metastasis in B16/F10.9 melanoma, 4T1 breast cancer, and EL4 thymoma mouse models $[117,118]$. Mechanistically the vaccination sensitizes fibroblasts to CD8 T-cell attack, which leads to decreased collagen production and the significantly increased uptake of chemotherapeutic drugs [119].

Stromal fibrosis can also be inhibited using antioxidants, such as high-dose vitamin $\mathrm{E}$, which increase survival in rat models [120] or by targeting angiotensin II type 1 receptors and angiotensin-converting enzyme activity [121]. In an anaplastic thyroid cancer xenograft model, bevacizumab (Avastin) treatment reduced the macrophage infiltration and inflammatory cytokine expression. More importantly, bevacizumab treatment reduced vascular permeability, thus affecting the first step of tumor stroma development [122]. There have so far been no convincing clinical studies showing the efficacy of tumor stroma inhibition in the most aggressive forms of thyroid cancer. However, because there is no treatment for the anaplastic form of thyroid cancer, studies of combination therapies targeting both the cancer cells and stroma might offer clinically relevant protocols and treatment alternatives.

Collectively, the continuously developing tumor stroma that simultaneously contains different stromal developmental phases has the versatile ability to support epithelial cancer cell growth. Although the presence and function of mesenchymal stem cells in the tumor microenvironment is still incompletely understood, the current knowledge suggests a crucial role for MSCs in the construction of the microenvironment, nurturing CSC/TIC cells, and supporting differentiated epithelial cancer cells. Based on this knowledge, targeting the stromal components in combination with the cancer cells themselves may increase the efficacy of cancer therapy.
Abbreviations
CAF: Cancer-associated fibroblast
CDC42: Cell division control protein 42 homolog
CSC: Cancer stem cell
CXCL12: Stromal derived factor 1 or SDF-1
CXCR4: C-X-C chemokine receptor type 4 or SDF-1 receptor
ECM: Extracellular matrix
FAK: $\quad$ Focal adhesion kinase
FAP: Fibroblast activation protein

GVHD: Graft-versus-host disease

HGF: Hepatocyte growth factor

HSV-TK: Herpes simplex virus-thymidine kinase

LOXL2: Lysyl oxidase-like 2

MMP: Matrix metalloproteinase

MSC: Mesenchymal stem cell

NO: $\quad$ Nitric oxide

PKC: $\quad$ Protein kinase C

ROBO1: Roundabout (axon guidance receptor)

ROCK: Rho-associated coiled-coil kinase

RNAi: RNA interference

ROS: Reactive oxygen species

RNS: Reactive nitrogen species

SLIT2: Slit homolog protein 2

SNAIL: Snail family zinc finger 1

TAF: Tumor-associated fibroblast

TIC: $\quad$ Tumor initiating cell

TWIST1: Twist family BHLH transcription factor I

VEGF: Vascular endothelial growth factor.

\section{Conflict of Interests}

The authors have no conflict of interests.

\section{References}

[1] E. R. Le Count, "Lymphoma, a benign tumor representing a lymph gland in structure," The Journal of Experimental Medicine, vol. 4, no. 5-6, pp. 559-567, 1899.

[2] I. Levin, "Changes in the tissue surrounding a growing tumor and the significance of the 'precancerous state"' Journal of Experimental Medicine, vol. 16, no. 2, pp. 149-154, 1912.

[3] I. Levin, "The mechanisms of metastasis formation in experimental cancer," Journal of Experimental Medicine, vol. 18, no. 4, pp. 397-405, 1913.

[4] S. O. Tamimi and A. Ahmed, "Stromal changes in invasive breast carcinoma: an ultrastructural study," Journal of Pathology, vol. 153, no. 2, pp. 163-170, 1987.

[5] D. Zipori, M. Krupsky, and P. Resnitzky, "Stromal cell effects on clonal growth of tumors," Cancer, vol. 60, no. 8, pp. 1757-1762, 1987.

[6] F. R. Miller, D. McEachern, and B. E. Miller, "Growth regulation of mouse mammary tumor cells in collagen gel cultures by diffusible factors produced by normal mammary gland epithelium and stromal fibroblasts," Cancer Research, vol. 49, no. 21, pp. 6091-6097, 1989.

[7] C. E. P. van Roozendaal, B. van Ooijen, J. G. M. Klijn et al., "Stromal influences on breast cancer cell growth," British Journal of Cancer, vol. 65, no. 1, pp. 77-81, 1992.

[8] X. Wu, C. Jin, F. Wang, C. Yu, and W. L. McKeehan, "Stromal cell heterogeneity in fibroblast growth factor-mediated stromalepithelial cell cross-talk in premalignant prostate tumors," Cancer Research, vol. 63, no. 16, pp. 4936-4944, 2003.

[9] M. Greenblatt and P. Shubi, "Tumor angiogenesis: transfilter diffusion studies in the hamster by the transparent chamber technique," Journal of the National Cancer Institute, vol. 41, no. 1, pp. 111-124, 1968.

[10] D. G. Duda, K. S. Cohen, S. V. Kozin et al., "Evidence for incorporation of bone marrow-derived endothelial cells into 
perfused blood vessels in tumors," Blood, vol. 107, no. 7, pp. 2774-2776, 2006.

[11] A. E. Woods and J. M. Papadimitriou, "The effect of inflammatory stimuli on the stroma of neoplasms: the involvement of mononuclear phagocytes," Journal of Pathology, vol. 123, no. 3, pp. 165-174, 1977.

[12] D. Lyden, K. Hattori, S. Dias et al., "Impaired recruitment of bone-marrow-derived endothelial and hematopoietic precursor cells blocks tumor angiogenesis and growth," Nature Medicine, vol. 7, no. 11, pp. 1194-1201, 2001.

[13] K. L. Andarawewa, E. R. Motrescu, M.-P. Chenard et al., "Stromelysin-3 is a potent negative regulator of adipogenesis participating to cancer cell-adipocyte interaction/crosstalk at the tumor invasive front," Cancer Research, vol. 65, no. 23, pp. 10862-10871, 2005.

[14] D. Bonnet and J. E. Dick, "Human acute myeloid leukemia is organized as a hierarchy that originates from a primitive hematopoietic cell," Nature Medicine, vol. 3, no. 7, pp. 730-737, 1997.

[15] S. K. Singh, C. Hawkins, I. D. Clarke et al., "Identification of human brain tumour initiating cells," Nature, vol. 432, no. 7015, pp. 396-401, 2004.

[16] A. Santamaria-Martínez, J. Barquinero, A. Barbosa-Desongles et al., "Identification of multipotent mesenchymal stromal cells in the reactive stroma of a prostate cancer xenograft by side population analysis," Experimental Cell Research, vol. 315, no. 17, pp. 3004-3013, 2009.

[17] G. van der Horst, L. Bos, and G. van der Pluijm, "Epithelial plasticity, cancer stem cells, and the tumor-supportive stroma in bladder carcinoma," Molecular Cancer Research, vol. 10, no. 8, pp. 995-1009, 2012.

[18] R. Liu, S. Wei, J. Chen, and S. Xu, "Mesenchymal stem cells in lung cancer tumor microenvironment: their biological properties, influence on tumor growth and therapeutic implications," Cancer letters, vol. 353, no. 2, pp. 145-152, 2014.

[19] A. Calon, E. Lonardo, A. Berenguer-Llergo et al., "Stromal gene expression defines poor-prognosis subtypes in colorectal cancer," Nature Genetics, vol. 47, pp. 320-329, 2015.

[20] C. Isella, A. Terrasi, S. E. Bellomo et al., "Stromal contribution to the colorectal cancer transcriptome," Nature Genetics, vol. 47, pp. 312-319, 2015.

[21] H. Choi, J. Sheng, D. Gao et al., "Transcriptome analysis of individual stromal cell populations identifies stroma-tumor crosstalk in mouse lung cancer model," Cell Reports, vol. 10, pp. 1187-1201, 2015.

[22] J. A. Nagy, L. F. Brown, D. R. Senger et al., "Pathogenesis of tumor stroma generation: a critical role for leaky blood vessels and fibrin deposition," Biochimica et Biophysica Acta-Reviews on Cancer, vol. 948, no. 3, pp. 305-326, 1989.

[23] I. Freitas, G. F. Baronzio, V. Bertone et al., "Stroma formation in Ehrlich carcinoma. I. Oedema phase. A mitosis burst as an index of physiological reoxygenation?" Anticancer Research, vol. 11, no. 2, pp. 569-578, 1991.

[24] H. F. Dvorak, V. S. Harvey, P. Estrella, L. F. Brown, J. McDonagh, and A. M. Dvorak, "Fibrin containing gels induce angiogenesis. Implications for tumor stroma generation and wound healing," Laboratory Investigation, vol. 57, no. 6, pp. 673-686, 1987.

[25] M. Löhr, C. Schmidt, J. Ringel et al., "Transforming growth factor-betal induces desmoplasia in an experimental model of human pancreatic carcinoma," Cancer Research, vol. 61, no. 2, pp. 550-555, 2001.
[26] J. A. Tuxhorn, G. E. Ayala, M. J. Smith, V. C. Smith, T. D. Dang, and D. R. Rowley, "Reactive stroma in human prostate cancer: induction of myofibroblast phenotype and extracellular matrix remodeling," Clinical Cancer Research, vol. 8, no. 9, pp. 29122923, 2002.

[27] H. F. Dvorak, N. S. Orenstein, A. C. Carvalho et al., "Induction of a fibrin-gel investment: an early event in line 10 hepatocarcinoma growth mediated by tumor-secreted products," Journal of Immunology, vol. 122, no. 1, pp. 166-174, 1979.

[28] E. Monaghan-Benson and K. Burridge, "The regulation of vascular endothelial growth factor-induced microvascular permeability requires Rac and reactive oxygen species," The Journal of Biological Chemistry, vol. 284, no. 38, pp. 25602-25611, 2009.

[29] C. DerMardirossian and G. M. Bokoch, "GDIs: central regulatory molecules in Rho GTPase activation," Trends in Cell Biology, vol. 15, no. 7, pp. 356-363, 2005.

[30] D. Fukumura, T. Gohongi, A. Kadambi et al., "Predominant role of endothelial nitric oxide synthase in vascular endothelial growth factor-induced angiogenesis and vascular permeability," Proceedings of the National Academy of Sciences of the United States of America, vol. 98, no. 5, pp. 2604-2609, 2001.

[31] D. B. McKay, " $\left[{ }^{3} \mathrm{H}\right]$ Noradrenaline accumulation in cultured bovine adrenal medullary cells: modulation of accumulation by nicotine," Naunyn-Schmiedeberg's Archives of Pharmacology, vol. 340, no. 6, pp. 610-616, 1989.

[32] D. Zhao, L. Qin, P.-M. Bourbon, L. James, H. F. Dvorak, and H. Zeng, "Orphan nuclear transcription factor TR3/Nur77 regulates microvessel permeability by targeting endothelial nitric oxide synthase and destabilizing endothelial junctions," Proceedings of the National Academy of Sciences of the United States of America, vol. 108, no. 29, pp. 12066-12071, 2011.

[33] C. A. Kunder, A. L. St John, and S. N. Abraham, "Mast cell modulation of the vascular and lymphatic endothelium," Blood, vol. 118, no. 20, pp. 5383-5393, 2011.

[34] H. F. Dvorak, “Tumors: wounds that do not heal. Similarities between tumor stroma generation and wound healing," The New England Journal of Medicine, vol. 315, no. 26, pp. 1650-1659, 1986.

[35] A. H. Klopp, L. Lacerda, A. Gupta et al., "Mesenchymal stem cells promote mammosphere formation and decrease ECadherin in normal and malignant breast cells," PLOS ONE, vol. 5, no. 8, Article ID e12180, 2010.

[36] H. F. Dvorak, J. A. Nagy, D. Feng et al., "Vascular permeability factor/vascular endothelial growth factor and the significance of microvascular hyperpermeability in angiogenesis," Current Topics in Microbiology and Immunology, vol. 237, pp. 98-132, 1999.

[37] M. Schmitt, F. Jänicke, N. Moniwa, N. Chucholowski, L. Pache, and H. Graeff, "Tumor-associated urokinase-type plasminogen activator: biological and clinical significance," Biological Chemistry Hoppe-Seyler, vol. 373, no. 7, pp. 611-622, 1992.

[38] E. Zorio, J. Gilabert-Estelles, F. Espana, L. A. Ramon, R. Cosin, and A. Estelles, "Fibrinolysis: the key to new pathogenetic mechanisms," Current Medicinal Chemistry, vol. 15, pp. 923929, 2008.

[39] J. A. Nagy, A. M. Dvorak, and H. F. Dvorak, "Vascular hyperpermeability, angiogenesis, and stroma generation," Cold Spring Harbor Perspectives in Medicine, vol. 2, no. 2, Article ID a006544, 2012.

[40] E. D’Asti, N. Magnus, B. Meehan, D. Garnier, and J. Rak, "Genetic basis of thrombosis in cancer," Seminars in Thrombosis and Hemostasis, vol. 40, no. 3, pp. 284-295, 2014. 
[41] H. F. Dvorak, A. M. Dvorak, E. J. Manseau, L. Wiberg, and W. H. Churchill, "Fibrin gel investment associated with line 1 and line 10 solid tumor growth, angiogenesis, and fibroplasia in guinea pigs. Role of cellular immunity, myofibroblasts, microvascular damage, and infarction in line 1 tumor regression," Journal of the National Cancer Institute, vol. 62, no. 6, pp. 1459-1472, 1979.

[42] L. VanDeWater, P. B. Tracy, D. Aronson, K. G. Mann, and H. F. Dvorak, "Tumor cell generation of thrombin via functional prothrombinase assembly," Cancer Research, vol. 45, no. 11, pp. 5521-5525, 1985.

[43] C. Yapijakis, A. Bramos, A. M. Nixon, V. Ragos, and E. Vairaktaris, "The interplay between hemostasis and malignancy: the oral cancer paradigm," Anticancer Research, vol. 32, no. 5, pp. 1791-1800, 2012.

[44] S. S. McAllister and R. A. Weinberg, "The tumour-induced systemic environment as a critical regulator of cancer progression and metastasis," Nature Cell Biology, vol. 16, no. 8, pp. 717-727, 2014.

[45] S. H. Barsky, C. N. Rao, G. R. Grotendorst, and L. A. Liotta, "Increased content of type $\mathrm{V}$ collagen in desmoplasia of human breast carcinoma," American Journal of Pathology, vol. 108, no. 3, pp. 276-283, 1982.

[46] O. Koperek, C. Scheuba, C. Puri et al., "Molecular characterization of the desmoplastic tumor stroma in medullary thyroid carcinoma," International Journal of Oncology, vol. 31, no. 1, pp. 59-67, 2007.

[47] C. Scheuba, K. Kaserer, K. Kaczirek, R. Asari, and B. Niederle, "Desmoplastic stromal reaction in medullary thyroid canceran intraoperative "marker" for lymph node metastases," World Journal of Surgery, vol. 30, no. 5, pp. 853-859, 2006.

[48] O. Koperek, R. Asari, B. Niederle, and K. Kaserer, "Desmoplastic stromal reaction in papillary thyroid microcarcinoma," Histopathology, vol. 58, no. 6, pp. 919-924, 2011.

[49] O. Koperek, E. Akin, R. Asari, B. Niederle, and N. Neuhold, "Expression of hypoxia-inducible factor 1 alpha in papillary thyroid carcinoma is associated with desmoplastic stromal reaction and lymph node metastasis," Virchows Archiv, vol. 463, no. 6, pp. 795-802, 2013.

[50] A. Toullec, D. Gerald, G. Despouy et al., "Oxidative stress promotes myofibroblast differentiation and tumour spreading," EMBO Molecular Medicine, vol. 2, no. 6, pp. 211-230, 2010.

[51] B. Z. Packard, V. V. Artym, A. Komoriya, and K. M. Yamada, "Direct visualization of protease activity on cells migrating in three-dimensions," Matrix Biology, vol. 28, no. 1, pp. 3-10, 2009.

[52] P. B. Gupta, T. T. Onder, G. Jiang et al., "Identification of selective inhibitors of cancer stem cells by high-throughput screening," Cell, vol. 138, no. 4, pp. 645-659, 2009.

[53] M. A. McNiven, "Breaking away: matrix remodeling from the leading edge," Trends in Cell Biology, vol. 23, no. 1, pp. 16-21, 2013.

[54] A. L. Jackson, R. Chen, and L. A. Loeb, "Induction of microsatellite instability by oxidative DNA damage," Proceedings of the National Academy of Sciences of the United States of America, vol. 95, no. 21, pp. 12468-12473, 1998.

[55] K. Ohuchida, K. Mizumoto, M. Murakami et al., "Radiation to stromal fibroblasts increases invasiveness of pancreatic cancer cells through tumor-stromal interactions," Cancer Research, vol. 64, no. 9, pp. 3215-3222, 2004.

[56] A. E. Karnoub, A. B. Dash, A. P. Vo et al., "Mesenchymal stem cells within tumour stroma promote breast cancer metastasis," Nature, vol. 449, no. 7162, pp. 557-563, 2007.
[57] S. Liu, C. Ginestier, S. J. Ou et al., "Breast cancer stem cells are regulated by mesenchymal stem cells through cytokine networks," Cancer Research, vol. 71, no. 2, pp. 614-624, 2011.

[58] K. McLean, Y. Gong, Y. Choi et al., "Human ovarian carcinomaassociated mesenchymal stem cells regulate cancer stem cells and tumorigenesis via altered BMP production," Journal of Clinical Investigation, vol. 121, no. 8, pp. 3206-3219, 2011.

[59] L. da Silva Meirelles, P. C. Chagastelles, and N. B. Nardi, "Mesenchymal stem cells reside in virtually all post-natal organs and tissues," Journal of Cell Science, vol. 119, no. 11, pp. 22042213, 2006.

[60] M. Studeny, F. C. Marini, R. E. Champlin, C. Zompetta, I. J. Fidler, and M. Andreeff, "Bone marrow-derived mesenchymal stem cells as vehicles for interferon- $\beta$ delivery into tumors," Cancer Research, vol. 62, no. 13, pp. 3603-3608, 2002.

[61] A. H. Klopp, E. L. Spaeth, J. L. Dembinski et al., “Tumor irradiation increases the recruitment of circulating mesenchymal stem cells into the tumor microenvironment," Cancer Research, vol. 67, no. 24, pp. 11687-11695, 2007.

[62] S. Kidd, E. Spaeth, J. L. Dembinski et al., "Direct evidence of mesenchymal stem cell tropism for tumor and wounding microenvironments using in vivo bioluminescent imaging," Stem Cells, vol. 27, no. 10, pp. 2614-2623, 2009.

[63] A. Sohni and C. M. Verfaillie, "Mesenchymal stem cells migration homing and tracking," Stem Cells International, vol. 2013, Article ID 130763, 8 pages, 2013.

[64] S. Lourenco, V. H. Teixeira, T. Kalber, R. J. Jose, R. A. Floto, and S. M. Janes, "Macrophage migration inhibitory factor-CXCR4 is the dominant chemotactic axis in human mesenchymal stem cell recruitment to tumors," The Journal of Immunology, vol. 194, pp. 3463-3474, 2015.

[65] E. L. Spaeth, J. L. Dembinski, A. K. Sasser et al., "Mesenchymal stem cell transition to tumor-associated fibroblasts contributes to fibrovascular network expansion and tumor progression," PLoS ONE, vol. 4, no. 4, Article ID e4992, 2009.

[66] G. Kharaishvili, D. Simkova, K. Bouchalova, M. Gachechiladze, N. Narsia, and J. Bouchal, "The role of cancer-associated fibroblasts, solid stress and other microenvironmental factors in tumor progression and therapy resistance," Cancer Cell International, vol. 14, no. 1, article no. 41, 2014.

[67] Y. Mao, E. T. Keller, D. H. Garfield, K. Shen, and J. Wang, "Stromal cells in tumor microenvironment and breast cancer," Cancer and Metastasis Reviews, vol. 32, no. 1-2, pp. 303-315, 2013.

[68] W. A. Schulz, M. Burchardt, and M. V. Cronauer, "Molecular biology of prostate cancer," Molecular Human Reproduction, vol. 9, no. 7-8, pp. 437-448, 2003.

[69] H. Luo, G. Tu, Z. Liu, and M. Liu, "Cancer-associated fibroblasts: a multifaceted driver of breast cancer progression," Cancer Letters, vol. 361, pp. 155-163, 2015.

[70] Y. P. Choi, J. H. Lee, M.-Q. Gao et al., "Cancer-associated fibroblast promote transmigration through endothelial brain cells in three-dimensional in vitro models," International Journal of Cancer, vol. 135, no. 9, pp. 2024-2033, 2014.

[71] R. Marlow, P. Strickland, J. S. Lee et al., "SLITs suppress tumor growth In vivo by silencing Sdfl/Cxcr4 within breast epithelium," Cancer Research, vol. 68, no. 19, pp. 7819-7827, 2008.

[72] A. Prasad, A. Z. Fernandis, Y. Rao, and R. K. Ganju, "Slit protein-mediated inhibition of CXCR4-induced chemotactic and chemoinvasive signaling pathways in breast cancer cells," The Journal of Biological Chemistry, vol. 279, no. 10, pp. 91159124, 2004. 
[73] P.-H. Chang, W. W. Hwang-Verslues, Y.-C. Chang et al., "Activation of Robol signaling of breast cancer cells by Slit2 from stromal fibroblast restrains tumorigenesis via blocking PI3K/Akt/beta-catenin pathway," Cancer Research, vol. 72, no. 18, pp. 4652-4661, 2012.

[74] M. C. Stella, L. Trusolino, and P. M. Comoglio, “The Slit/Robo system suppresses hepatocyte growth factor-dependent invasion and morphogenesis," Molecular Biology of the Cell, vol. 20, no. 2, pp. 642-657, 2009.

[75] A. Takahashi, G. Ishii, S. Neri et al., "Podoplanin-expressing cancer-associated fibroblasts inhibit small cell lung cancer growth," Oncotarget, vol. 6, pp. 9531-9541, 2015.

[76] K. Anton, D. Banerjee, and J. Glod, "Macrophage-associated mesenchymal stem cells assume an activated, migratory, proinflammatory phenotype with increased IL-6 and CXCL10 secretion," PLoS ONE, vol. 7, no. 4, Article ID e35036, 2012.

[77] R. S. Waterman, S. L. Tomchuck, S. L. Henkle, and A. M. Betancourt, "A new mesenchymal stem cell (MSC) paradigm: polarization into a pro-inflammatory $\mathrm{MSCl}$ or an immunosuppressive MSC2 phenotype," PLoS ONE, vol. 5, no. 4, Article ID e10088, 2010

[78] L. Lacerda, B. G. Debeb, D. Smith et al., "Mesenchymal stem cells mediate the clinical phenotype of inflammatory breast cancer in a preclinical model," Breast Cancer Research, vol. 17, article 42, 2015.

[79] M. Di Nicola, C. Carlo-Stella, M. Magni et al., "Human bone marrow stromal cells suppress T-lymphocyte proliferation induced by cellular or nonspecific mitogenic stimuli," Blood, vol. 99, no. 10, pp. 3838-3843, 2002.

[80] R. Ramasamy, E. W.-F. Lam, I. Soeiro, V. Tisato, D. Bonnet, and F. Dazzi, "Mesenchymal stem cells inhibit proliferation and apoptosis of tumor cells: impact on in vivo tumor growth," Leukemia, vol. 21, no. 2, pp. 304-310, 2007.

[81] M. Takeda, T. Mikami, Y. Numata, M. Okamoto, and I. Okayasu, "Papillary thyroid carcinoma with heterotopic ossification is a special subtype with extensive progression," American Journal of Clinical Pathology, vol. 139, no. 5, pp. 587-598, 2013.

[82] H. Kuribayashi, K. Tsuta, E. Mizutani et al., "Clinicopathological analysis of primary lung carcinoma with heterotopic ossification," Lung Cancer, vol. 64, no. 2, pp. 160-165, 2009.

[83] M. N. Rumi, G. S. Deol, K. P. Singapuri, and V. D. Pellegrini Jr., "The origin of osteoprogenitor cells responsible for heterotopic ossification following hip surgery: an animal model in the rabbit," Journal of Orthopaedic Research, vol. 23, no. 1, pp. 3440, 2005.

[84] C. A. O’Brien, A. Pollett, S. Gallinger, and J. E. Dick, “A human colon cancer cell capable of initiating tumour growth in immunodeficient mice," Nature, vol. 445, no. 7123, pp. 106-110, 2007.

[85] S. A. Patel, M. A. Dave, S. A. Bliss et al., “T ${ }_{\text {reg }} /$ Th17 polarization by distinct subsets of breast cancer cells is dictated by the interaction with mesenchymal stem cells," Journal of Cancer Stem Cell Research, vol. 2, Article ID e1003, 2014.

[86] B. G. Cuiffo, A. Campagne, G. W. Bell et al., "MSC-regulated microRNAs converge on the transcription factor FOXP2 and promote breast cancer metastasis," Cell Stem Cell, vol. 15, no. 6, pp. 762-774, 2014.

[87] K. Denzer, M. J. Kleijmeer, H. F. G. Heijnen, W. Stoorvogel, and H. J. Geuze, "Exosome: from internal vesicle of the multivesicular body to intercellular signaling device," Journal of Cell Science, vol. 113, part 19, pp. 3365-3374, 2000.
[88] S. Atay, S. Banskota, J. Crow, G. Sethi, L. Rink, and A. K. Godwin, "Oncogenic KIT-containing exosomes increasegastrointestinal stromal tumor cell invasion," Proceedings of the National Academy of Sciences of the United States of America, vol. 111, no. 2, pp. 711-716, 2014.

[89] K. C. Vallabhaneni, H. Haller, and I. Dumler, "Vascular smooth muscle cells initiate proliferation of mesenchymal stem cells by mitochondrial transfer via tunneling nanotubes," Stem Cells and Development, vol. 21, no. 17, pp. 3104-3113, 2012.

[90] V. Luga, L. Zhang, A. M. Viloria-Petit et al., "Exosomes mediate stromal mobilization of autocrine Wnt-PCP signaling in breast cancer cell migration," Cell, vol. 151, no. 7, pp. 1542-1556, 2012.

[91] P. K. Lim, S. A. Bliss, S. A. Patel et al., "Gap junction-mediated import of microRNA from bone marrow stromal cells can elicit cell cycle quiescence in breast cancer cells," Cancer Research, vol. 71, no. 5, pp. 1550-1560, 2011.

[92] M. D. Milsom and A. Trumpp, "Bridging the information gap," Nature Immunology, vol. 12, no. 5, pp. 377-379, 2011.

[93] E. T. Ishikawa, D. Gonzalez-Nieto, G. Ghiaur et al., "Connexin43 prevents hematopoietic stem cell senescence through transfer of reactive oxygen species to bone marrow stromal cells," Proceedings of the National Academy of Sciences of the United States of America, vol. 109, no. 23, pp. 9071-9076, 2012.

[94] G. Rappa, J. Mercapide, and A. Lorico, "Spontaneous formation of tumorigenic hybrids between breast cancer and multipotent stromal cells is a source of tumor heterogeneity," The American Journal of Pathology, vol. 180, no. 6, pp. 2504-2515, 2012.

[95] J. L. Spees, S. D. Olson, M. J. Whitney, and D. J. Prockop, "Mitochondrial transfer between cells can rescue aerobic respiration," Proceedings of the National Academy of Sciences of the United States of America, vol. 103, no. 5, pp. 1283-1288, 2006.

[96] S. Strassburg, N. W. Hodson, P. I. Hill, S. M. Richardson, and J. A. Hoyland, "Bi-directional exchange of membrane components occurs during co-culture of mesenchymal stem cells and nucleus pulposus cells," PLoS ONE, vol. 7, no. 3, Article ID e33739, 2012.

[97] M. D. Castellone, L. E. Laatikainen, J. P. Laurila et al., "Brief report: mesenchymal stromal cell atrophy in coculture increases aggressiveness of transformed cells," Stem Cells, vol. 31, no. 6, pp. 1218-1223, 2013.

[98] K. Mandel, Y. Yang, A. Schambach, S. Glage, A. Otte, and R. Hass, "Mesenchymal stem cells directly interact with breast cancer cells and promote tumor cell growth in vitro and in vivo," Stem Cells and Development, vol. 22, no. 23, pp. 3114-3127, 2013.

[99] M. Pietilä, P. Lehenkari, P. Kuvaja et al., "Mortalin antibodyconjugated quantum dot transfer from human mesenchymal stromal cells to breast cancer cells requires cell-cell interaction," Experimental Cell Research, vol. 319, no. 18, pp. 2770-2780, 2013.

[100] M. Abercrombie, "The bases of the locomotory behaviour of fibroblasts," Experimental Cell Research, vol. 8, pp. 188-198, 1961.

[101] H. Eagle, G. E. Foley, H. Koprowski, H. Lazarus, E. M. Levine, and R. A. Adams, "Growth characteristics of virus-transformed cells. Maximum population density, inhibition by normal cells, serum requirement, growth in soft agar, and xenogeneic transplantability," The Journal of Experimental Medicine, vol. 131, no. 4, pp. 863-879, 1970.

[102] E. I. Azzam, S. M. de Toledo, and J. B. Little, "Direct evidence for the participation of gap junction-mediated intercellular communication in the transmission of damage signals from $\alpha$-particle irradiated to nonirradiated cells," Proceedings of the National Academy of Sciences of the United States of America, vol. 98, no. 2, pp. 473-478, 2001. 
[103] Q. Huang, F. Li, X. Liu et al., "Caspase 3-mediated stimulation of tumor cell repopulation during cancer radiotherapy," Nature Medicine, vol. 17, no. 7, pp. 860-866, 2011.

[104] A. H. Klopp, A. Gupta, E. Spaeth, M. Andreeff, and F. Marini III, "Concise review: dissecting a discrepancy in the literature: do mesenchymal stem cells support or suppress tumor growth?" Stem Cells, vol. 29, no. 1, pp. 11-19, 2011.

[105] H. Niess, J. C. von Einem, M. N. Thomas et al., "Treatment of advanced gastrointestinal tumors with genetically modified autologous mesenchymal stromal cells (TREAT-ME1): study protocol of a phase I/II clinical trial," BMC Cancer, vol. 15, article 237, 2015.

[106] F. Baron, C. Lechanteur, E. Willems et al., "Cotransplantation of mesenchymal stem cells might prevent death from graftversus-host disease (GVHD) without abrogating graft-versustumor effects after HLA-mismatched allogeneic transplantation following nonmyeloablative conditioning," Biology of Blood and Marrow Transplantation, vol. 16, pp. 838-847, 2010.

[107] J. Kurtzberg, S. Prockop, P. Teira et al., "Allogeneic human mesenchymal stem cell therapy (remestemcel-L, Prochymal) as a rescue agent for severe refractory acute graft-versus-host disease in pediatric patients," Biology of Blood and Marrow Transplantation, vol. 20, no. 2, pp. 229-235, 2014.

[108] M. L. MacMillan, B. R. Blazar, T. E. DeFor, and J. E. Wagner, "Transplantation of ex-vivo culture-expanded parental haploidentical mesenchymal stem cells to promote engraftment in pediatric recipients of unrelated donor umbilical cord blood: results of a phase I-II clinical trial," Bone Marrow Transplantation, vol. 43, no. 6, pp. 447-454, 2009.

[109] H. M. Lazarus, O. N. Koc, S. M. Devine et al., "Cotransplantation of HLA-identical sibling culture-expanded mesenchymal stem cells and hematopoietic stem cells in hematologic malignancy patients," Biology of Blood and Marrow Transplantation, vol. 11, no. 5, pp. 389-398, 2005.

[110] S. Corso and S. Giordano, "Cell-autonomous and non-cellautonomous mechanisms of HGF/MET-driven resistance to targeted therapies: from basic research to a clinical perspective," Cancer Discovery, vol. 3, no. 9, pp. 978-992, 2013.

[111] K. P. Olive, M. A. Jacobetz, C. J. Davidson et al., "Inhibition of Hedgehog signaling enhances delivery of chemotherapy in a mouse model of pancreatic cancer," Science, vol. 324, no. 5933, pp. 1457-1461, 2009.

[112] G. L. Beatty and Y. Paterson, "IFN- $\gamma$ can promote tumor evasion of the immune system in vivo by down-regulating cellular levels of an endogenous tumor antigen," The Journal of Immunology, vol. 165, no. 10, pp. 5502-5508, 2000.

[113] M. Mersmann, A. Schmidt, J. F. Rippmann et al., "Human antibody derivatives against the fibroblast activation protein for tumor stroma targeting of carcinomas," International Journal of Cancer, vol. 92, no. 2, pp. 240-248, 2001.

[114] R.-D. Hofheinz, S.-E. Al-Batran, F. Hartmann et al., "Stromal antigen targeting by a humanised monoclonal antibody: an early phase II trial of sibrotuzumab in patients with metastatic colorectal cancer," Onkologie, vol. 26, no. 1, pp. 44-48, 2003.

[115] A. M. Scott, G. Wiseman, S. Welt et al., "A phase I doseescalation study of sibrotuzumab in patients with advanced or metastatic fibroblast activation protein-positive cancer," Clinical Cancer Research, vol. 9, no. 5, pp. 1639-1647, 2003.

[116] P. C. Schuberth, C. Hagedorn, S. M. Jensen et al., "Treatment of malignant pleural mesothelioma by fibroblast activation protein-specific re-directed T cells," Journal of Translational Medicine, vol. 11, no. 1, article 187, 11 pages, 2013.
[117] J. Lee, M. Fassnacht, S. Nair, D. Boczkowski, and E. Gilboa, "Tumor immunotherapy targeting fibroblast activation protein, a product expressed in tumor-associated fibroblasts," Cancer Research, vol. 65, no. 23, pp. 11156-11163, 2005.

[118] S. Gottschalk, F. Yu, M. Ji, S. Kakarla, and X.-T. Song, "A vaccine that co-targets tumor cells and cancer associated fibroblasts results in enhanced antitumor activity by inducing antigen spreading," PLoS ONE, vol. 8, no. 12, Article ID e82658, 2013.

[119] M. Loeffler, J. A. Krüger, A. G. Niethammer, and R. A. Reisfeld, "Targeting tumor-associated fibroblasts improves cancer chemotherapy by increasing intratumoral drug uptake," The Journal of Clinical Investigation, vol. 116, no. 7, pp. 1955-1962, 2006.

[120] X. C. Li, X. L. Lu, and H. H. Chen, " $\alpha$-Tocopherol treatment ameliorates chronic pancreatitis in an experimental rat model induced by trinitrobenzene sulfonic acid," Pancreatology, vol. 11, no. 1, pp. 5-11, 2011.

[121] A. Kuno, T. Yamada, K. Masuda et al., "Angiotensin-converting enzyme inhibitor attenuates pancreatic inflammation and fibrosis in male Wistar Bonn/Kobori rats," Gastroenterology, vol. 124, no. 4, pp. 1010-1019, 2003.

[122] A. V. Salnikov, N.-E. Heldin, L. B. Stuhr et al., "Inhibition of carcinoma cell-derived VEGF reduces inflammatory characteristics in xenograft carcinoma," International Journal of Cancer, vol. 119, no. 12, pp. 2795-2802, 2006. 

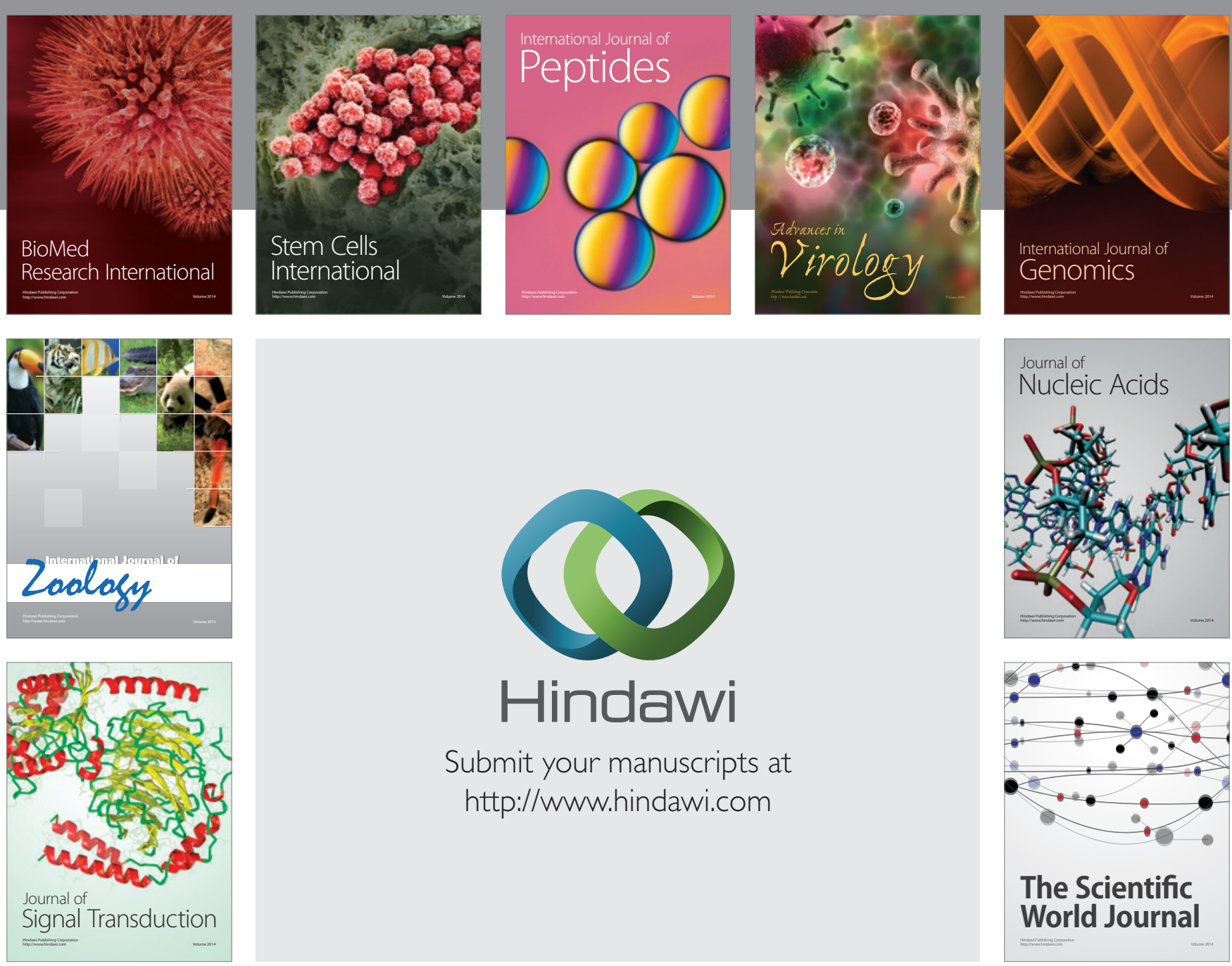

Submit your manuscripts at

http://www.hindawi.com
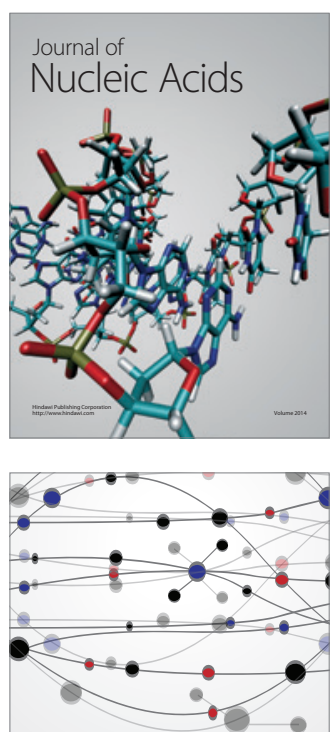

The Scientific World Journal
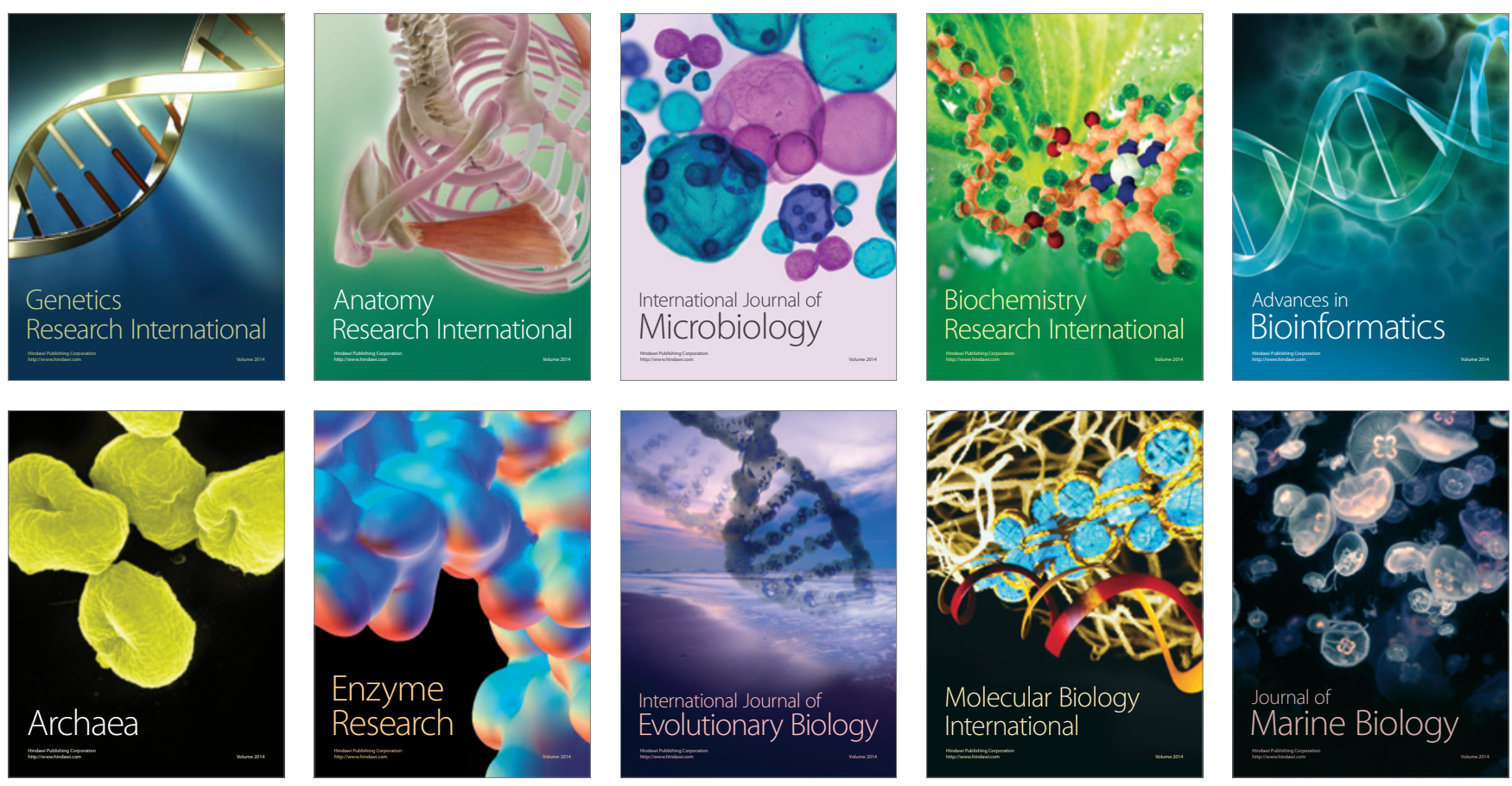